\title{
Microwave Transmission Through One-Dimensional Hybrid Quasi-Regular (Fibonacci and Thue-Morse)/ Periodic Structures
}

\author{
Youssef TRABELSI*, Naim BENALI, Yassine BOUAZZI, and Mounir KANZARI \\ LPMS Laboratory of Photovoltaic and Semi-conductor Materials, ENIT, EL Manar University, ENIT-BP 37, Belvédère \\ 1002 Tunis, Tunisia \\ *Corresponding author: Youssef TRABELSI_Ｅ-mail: youssef_trabelsi@yahoo.fr
}

\begin{abstract}
The transmission properties of hybrid quasi-periodic photonic systems (HQPS) made by the combination of one-dimensional periodic photonic crystals (PPCs) and quasi-periodic photonic crystals (QPCs) were theoretically studied. The hybrid quasi-periodic photonic lattice based on the hetero-structures was built from the Fibonacci and Thue-Morse sequences. We addressed the microwave properties of waves through the one-dimensional symmetric Fibonacci, and Thue-Morse system i.e., a quasi-periodic structure was made up of two different dielectric materials (Rogers and air), in the quarter wavelength condition. It shows that controlling the Fibonacci parameters permits to obtain selective optical filters with the narrow passband and polychromatic stop band filters with varied properties which can be controlled as desired. From the results, we presented the self-similar features of the spectra, and we also presented the fractal process through a return map of the transmission coefficients. We extracted powerfully the band gaps of hybrid quasi-periodic multilayered structures, called "pseudo band gaps", often containing resonant states, which could be considered as a manifestation of numerous defects distributed along the structure. The results of transmittance spectra showed that the cutoff frequency could be manipulated through the thicknesses of the defects and the type of dielectric layers of the system. Taken together, the above two properties provide favorable conditions for the design of an all-microwave intermediate reflector.
\end{abstract}

Keywords: Photonic crystals, aperiodic sequence, quasi-periodic photonic band gap, Thue-Morse class quasi-periodic, Fibonacci class quasi-periodic microwave reflector

Citation: Youssef TRABELSI, Naim BENALI, Yassine BOUAZZI, and Mounir KANZARI, "Microwave Transmission Through One-Dimensional Hybrid Quasi-Regular (Fibonacci and Thue-Morse)/Periodic Structures," Photonic Sensors, DOI: 10.1007/s13320-013-0114-7.

\section{Introduction}

Photonic crystals (PCs) are artificial materials made from periodic arrays of dielectric or metallic building blocks. The existence of photonic band gaps (PBGs) has brought about an unprecedented power to control and manipulate the propagation of electromagnetic (EM) waves [1-3]. The simplest form of a photonic crystal is the one-dimensional periodic structure. It is known as the Bragg mirror. It consists of a stack of alternating layers having a low and a high refractive indices and a thickness in the order of $\lambda / 4$ where $\lambda$ is the reference wavelength (i.e. whose thicknesses satisfy the Bragg condition). In

Received: 5 April 2013 / Revised version: 19 May 2013

(C) The Author(s) 2013. This article is published with open access at Springerlink.com 
contrast to the two- and three-dimensional cases, the one-dimensional photonic crystal is attractive since its production is more feasible at any wavelength scale.

The first function of a PBG is to be an excellent reflective mirror (more than 99\%). Therefore, one option for providing a more flexible design of band gap structures could be to increase the width of the PBGs. For this purpose, typically the optical contrast or the number of periods of the regular structure can increase. However, other methods could be proposed to enhance the photonic band gap. These could be based on the quasi-periodic sequence insertion. Furthermore, the introduction of quasi-periodic lattices to photonic crystals offers the prospect to carry out microwave or electromagnetic devices able to store, guide and filter the wave [4-6].

A quasi-crystal (QC) does not have a geometrical periodicity but is still deterministically generated: this is the reason why it possesses a Fibonacci sequence which is intermediate between a periodic structure and a random structure [7].

Thue-Morse (T-M) and Fibonacci systems are the most common examples of one-dimensional quasi-crystal. Recently, not only periodic structures or PCs with defects, but also quasi-periodic systems (QPs) have become significant structures of photonic crystals. Perfectly periodic and random structures cover only the two extremes of the rich spectrum of complex dielectric structures. These structures do not possess a translational symmetry and illustrate some remarkable physical properties such as the band gaps, resonance frequencies, and high localized states [8].

The quasi-one-dimensional aperiodic systems are multilayer structures composed of at least two different substrates (homogenous layers) $\mathrm{H}$ and $\mathrm{L}$ distributed along the given direction. The $\mathrm{H}$ and $\mathrm{L}$ layers alignment is precisely defined by the substitution rules or inflation scheme $[9,10]$. $\mathrm{H}$ denotes the material with the higher refractive index, and $\mathrm{L}$ denotes the one with the lower refractive index.
The transmission of the wave through the quasi-periodic multilayer arranged by the Fibonacci or Thue-Morse sequences has been widely studied in different domains [11]. It can be applied for special applications such as the possibility of optical frequency-selective filters and memories.

This quasi-periodic system (QPS) introduces a tapered periodicity responsible for forming a multitude of gaps, where the electromagnetic wave is forbidden in each frequency, and form reflector systems. It can be classified according to different criteria divided into two broad categories: namely self-similar and non-self-similar ones.

The different properties of quasi-periodic lattices can be exploited to design and build the ultra-compact modulate reflector and cavities resonant in all specific frequency domains.

The introduction of some defects to the quasi-periodic structure provides a novel arrangement of layers which exibit an association of defects inserted by the quasi-periodic lattice and the intrinsic defects locally inside the structure. Furthermore, the radiation frequencies corresponding to the quasi-photonic band gap are restricted to a defect that products a local field fascinated inside the defect because the propagation is forbidden inside the quasi-crystal.

So, a main attraction of various applications is based on the controlled insertion of defects within the crystal at the time of its manufacture in order to make the forbidden band as wide as possible to confine the light of any wavelengths $[12,13]$.

Moreover, the manufacture of photonic crystals is still a real challenge of the microwave domain, and undesirable defects can appear leading to a essential study of the charity of the periodicity for new devices [14-17].

The insertion of the defects into the quasi-periodic multilayer photonic crystals is based on the frequency shift and distortion of the transmission spectrum, where the frequency of the defect mode is very sensitive to the defect layer 
position as well as its thickness.

In this paper, the transmission spectra through the photonic band gap material with the quasi-crystal order according to hybrid generalized quasi-periodic sequences are examined in the microwave domain. The combination of the configuration quasi-periodic shapes: [(FIB $(m, n) /$ Defect/ FIB $(m, n)]$ and $[(\mathrm{TM}(m, n) / \operatorname{Defect} /(\mathrm{TM}(m$, $n)$ ], forms an effective hybrid photonic crystal (HPC) filter, where $m$ and $n$ are the parameters of quasi-periodic multilayered stacks, respectively. The transmission spectra of these novel linear hybrid quasi-periodic photonic crystal possess a multitude of band gaps split by harmonic spectral or transmission peaks. The positions of resonator peaks are dependent on the type of distribution layers. The important characteristics of the quasi-periodic order is a transmission spectrum varying according to the arrangement of layers. This transmission spectrum is controlled by the parameters of the quasi-periodic sequences due to their peculiar fractal gaps explained by the different interface correlations.

Consequently, an association of the high reflector with multitude narrow band gaps can be easily designed, which can be used for photonic applications.

\section{Formulation method}

Firstly, we considered the propagation of electromagnetic waves in a simple quasi-periodic layered media consisting of two possibilities: layers of different refractive indices or layers of similar refractive indices, and assumed that the material is non-magnetic. We employed the transfer matrix method (TMM) [18] in order to study the reflection properties of the quasi-periodic photonic crystal to extract the reflection and transmission spectrum through this system.

The TMM approach technique is a finite difference method particularly well suitable for the study of PBG materials, which can solve the standard problem of the photonic band gap structures and the scattering (transmission, reflection, and absorption) spectrum [18].

It is based on the Abeles method in terms of forward and backward propagating electric fields, $E_{0}^{+}$and $E_{0}^{-}$, which are introduced to calculate the reflection and transmission. Abeles showed that the relation between the amplitudes [18] of the electric fields of the incident wave $E_{0}^{+}$, reflected wave $E_{0}^{-}$, and transmitted wave after $m$ layers, $E_{m+1}^{+}$, is expressed as the following matrix for stratified films within $m$ layers:

$$
\left(\begin{array}{l}
E_{0}^{+} \\
E_{0}^{-}
\end{array}\right)=\frac{C_{1} C_{2} C_{3} \ldots C_{m+1}}{t_{1} t_{2} t_{3} \ldots t_{m+1}}\left(\begin{array}{l}
E_{m+1}^{+} \\
E_{m+1}^{-}
\end{array}\right)
$$

where $C_{j}$ is the propagation matrix with the matrix elements given by the following expression:

$$
C_{j}=\left(\begin{array}{cc}
\exp \left(i \varphi_{j-1}\right) & r_{j} \exp \left(-i \varphi_{j-1}\right) \\
r_{j} \exp \left(i \varphi_{j-1}\right) & \exp \left(-i \varphi_{j-1}\right)
\end{array}\right)
$$

where $t_{j}$ and $r_{j}$ are the Fresnel transmission and reflection coefficients, respectively, between the $(j-1)$ th and $j$ th layers.

The values $\varphi_{j-1}$ appearing in the expression of the propagation matrix $C_{j}$ indicate the shift in the phase between considered $(j-1)$ th and $j$ th boundaries, which are expressed by

$$
\varphi_{j-1}=\frac{2 \pi}{\lambda} \hat{n}_{j-1} d_{j-1} \cos \theta_{j-1} ; \varphi_{0}=0
$$

where $\lambda$ defines the wavelength of the incident light in the vacuum, and $d_{j-1}$ is the thickness of the $(j-1)$ th layer.

The Fresnel coefficients $t_{j}$ and $r_{j}$ can be expressed as follows by using the complex refractive index $\hat{n}_{j}=n_{j}+i k_{j}$ and the complex refractive angle $\theta_{j}$.

For the parallel (p) polarization:

$$
\begin{aligned}
r_{j p} & =\frac{\hat{n}_{j-1} \cos \theta_{j}-\hat{n}_{j} \cos \theta_{j-1}}{\hat{n}_{j-1} \cos \theta_{j}+\hat{n}_{j} \cos \theta_{j-1}} \\
t_{j p} & =\frac{2 \hat{n}_{j-1} \cos \theta_{j-1}}{\hat{n}_{j-1} \cos \theta_{j}+\hat{n}_{j} \cos \theta_{j-1}} .
\end{aligned}
$$

The complex refractive indices and the complex angles of the incidence obviously follow the Snell's law: $\hat{n}_{j-1} \sin \theta_{j-1}=\hat{n}_{j} \sin \theta_{j}$ with $j=[1, m+1]$. 
The incident wave is polarized with its electric field perpendicular to the plane described above. The light is said to be s-polarized (perpendicular). For the s-polarized light, the reflection coefficient is given by

$$
R_{j s}=\left|\frac{\hat{n}_{j-1} \cos \theta_{j-1}-\hat{n}_{j} \cos \theta_{j}}{\hat{n}_{j-1} \cos \theta_{j}+\hat{n}_{j} \cos \theta_{j-1}}\right|^{2}
$$

where the second form is derived from the first by eliminating $\theta_{j}$ using the Snell's law and trigonometric identities.

For the p-polarized light, the $R$ is given by

$$
R_{j p}=\left|\frac{\hat{n}_{j-1} \cos \theta_{j}-\hat{n}_{j} \cos \theta_{j-1}}{\hat{n}_{j-1} \cos \theta_{j}+\hat{n}_{j} \cos \theta_{j-1}}\right|^{2} .
$$

As a consequence of the conservation of energy, the transmission coefficients are given by [18].

For s- and p-polarizations, and the energy transmittance $\mathrm{T}$ is

$$
\begin{gathered}
T_{s}=\operatorname{Re}\left(\frac{\hat{n}_{m+1} \cos \theta_{m+1}}{\hat{n}_{0} \cos \theta_{0}}\right)\left|t_{s}\right|^{2} \\
T_{p}=\operatorname{Re}\left(\frac{\cos \theta_{m+1} / \hat{n}_{m+1}}{\cos \theta_{0} / \hat{n}_{0}}\right)\left|\frac{\hat{n}_{m+1}}{\hat{n}_{0}} t_{s}\right|^{2}
\end{gathered}
$$

where $t_{s}$ is the total transmission coefficient for perpendicular (s) polarization.

By setting $E_{m+1}^{-}=1$, because there is no reflection from the final phase, Abeles obtained a convenient formula for the total reflection and transmission coefficients, which correspond to the amplitude reflectance $r$ and transmittance $t$, respectively, as follows:

$$
r=\frac{E_{0}^{-}}{E_{0}^{+}}=\frac{c}{a} ; t=\frac{E_{m+1}^{+}}{E_{0}^{+}}=\frac{t_{1} t_{2} \ldots t_{m+1}}{a} .
$$

The quantities $a$ and $c$ are the matrix elements of the all product $C_{j}$ matrix.

\section{Generalized quasi-periodic Fibonacci model}

The one-dimensional Fibonacci sequence is a well-known example of the quasi-periodic system, which can be generated by using the substitution rule: $\mathrm{H} \rightarrow \mathrm{HL}$ and $\mathrm{L} \rightarrow \mathrm{H}$, where, $\mathrm{H}$ and $\mathrm{L}$ are defined as homogeneous ideal dielectric layers with different widths and the refractive indices, and these parameters are degrees of the freedom for manipulation of the reflection and transmission characteristics.

Thus starting with the single line segment $\mathrm{H}$ and repeating the deflation, a Fibonacci chain containing an arbitrarily large number of points can be generated.

The generalized Fibonacci sequence is a class of the quasi-periodic lattice generated by substitution rules as: $\mathrm{H} \rightarrow \mathrm{H}^{m} \mathrm{~L}^{n}$ and $\mathrm{L} \rightarrow \mathrm{H}^{m}$, where $m, n$ are the positive integer number and generalized Fibonacciclass model factor.

Figure 1 shows an example of the onedimensional generalized Fibonacci-class (GFC) for corresponding parameters $(k=4, m=2, n=2)$ and with normal incident light in the $z$-direction. For the construction of the Fibonacci sequence, two basic elements named $\mathrm{H}$ and $\mathrm{L}$ are necessary with arbitrary positive integers $m$ and $n$, where FS $(1,1)$ corresponds to the well-known standard Fibonacci sequence.

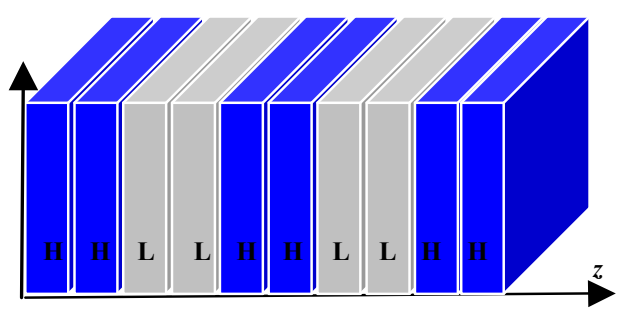

Fig. 1 Distribution of the multilayer's stack through the generalized Fibonacci sequence for the considered parameters of the Fibonacci sequence $(m=2, n=2)$.

Equivalently, they can also be generated by the substitution rule.

The total number of letters $\mathrm{H}$ and $\mathrm{L}$ in the word $S_{k+1}$ is denoted by $F_{k+1}$ and satisfies the recursion relation: $F_{k+1}=m F_{k}+n F_{k-1}$, where $F_{1}=F_{0}=1$. In the limit of an infinite sequence, the ratio of word lengths for subsequent inflation steps is given by

$$
\sigma=\lim _{k \rightarrow \infty} \frac{F_{k+1}}{F_{k}}=+\frac{m+\sqrt{m^{2}+4 n}}{2} .
$$


Also, one can show that the following recursion relation is satisfied for the Fibonacci structure as

$$
S_{k+1}=S_{k}^{m} S_{k-1}^{n} \text {. }
$$

For exemple, the multilayer stack distributed according the 3rd order of the generalized Fibonacci sequence and for $m=n=2$ is: $S_{3}=\left(S_{2}^{2} S_{1}^{2}\right)=$ $(\mathrm{HHBB})^{2}(\mathrm{H})^{2}$.

\section{Generalized quasi-periodic Thue-Morse model}

One-dimensional quasi-periodic Thue-Morse sequences are multilayer structures consisting of two different materials based on the two-letter alphabet $(\mathrm{H}, \mathrm{L})$ and the substitution rule $\sigma(\mathrm{H})=\mathrm{LH}, \sigma(\mathrm{H})=$ LH. The generalized Thue-Morse multilayer is recursively constructed as $S_{k+1}=S_{k}^{m} \bar{S}_{k-1}^{n}, \overline{S_{0}}=$ L and $S_{0}=\mathrm{H}, k$ is the order of the Thue-Morse sequence, $m$ and $n$ are positive integers, and $\bar{S}$ is the complementary sequence of $S$.

Based on the characteristics of the construction of the Thue-Morse sequence, Fig. 2 shows the one-dimensional generalized Thue-Morse class quasi-periodic multilayer stacks for 3rd generation of the Thue-Morse sequence. According to the Thue-Morse rule, there are 16 layers in this structure.

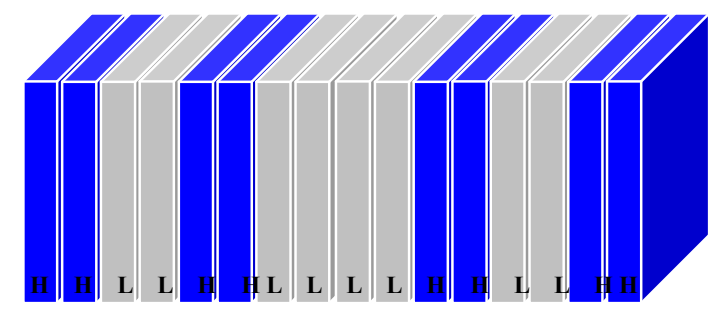

Fig. 2 Schematic representation showing the geometry of the 3rd generation of the generalized Thue-Morse (GTM) quasiperiodic multilayer system for $m=n=2$.

\section{Results and simulation}

In the following numerical investigation, we choose air $(L)$ and Roger $(H)$ as two elementary layers, with refractive indices $n_{L}=1$ and $n_{H}=3.19$, respectively. The thicknesses $d_{L, H}$ of the two materials have been chosen to satisfy the Bragg condition: $d_{L} n_{L}=d_{H} n_{H}=\lambda_{0} / 4$. We used the transfer matrix method to extract the transmission coefficients in the spectral range $0-50 \mathrm{GHz}$.

We showed that the corresponding transmission coefficients exhibited interesting properties, so it was found that the reflection bands of the Fibonacci sequence structures could cover all the spectral range by increasing the two parameters $m=n$. The Fibonacci sequences are taken $S_{k+1}=S_{k}^{m} S_{k-1}^{n}$. As shown in Fig. 3, the transmission spectrum of the generalized Fibonacci sequence contains several stop band gaps. Such arrangements of the narrow stop band gaps are repeated periodically with respect to $\lambda$. When increasing the parameter $m$ and $n$ of the generalized Fibonacci sequence $\operatorname{GF}(m, n)$, the photonic band gaps are split, and very narrow transmission peaks appear. The size of every band gap for the corresponding distribution $m=n=2$ or $m=n=3$ increase gradually versus the frequencies $(\mathrm{GHz})$. Also, the amplitude of the corresponding oscillation around each band gap increases with the width of band gaps.

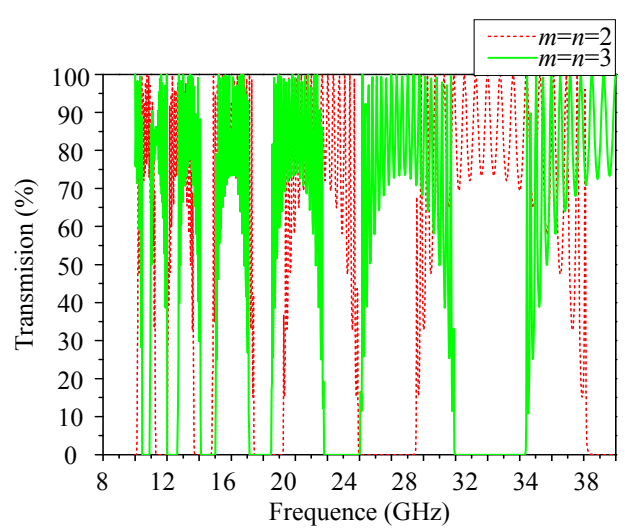

Fig. 3 Appearance of multiple PBGs of the transmission spectrum from the generalized Fibonacci sequence, $m=n=2$ and $m=n=3$, respectively.

The corresponding transmission from the quasi-periodic multilayer's stack arranged by the generalized Fibonacci sequence exhibits several zero-transmissions versus the parameters of quasi-periodic sequences $m$ and $n$, where the propagation of the electromagnetic wave in each forbidden gap is inhibited. The position of frequency 
centers $\left[F_{C}(\mathrm{GHz})\right]$ of considered pseudo band gaps depends on the number of layers and the form of the distribution. The position of centers of pseudo PBGs shifts from lower frequencies by increasing the parameter $p$, where $p=m=n$, and satisfies approximately the following law:

$$
F_{C}(p)=23 p e^{\frac{-23 p}{5}}+22 \text {. }
$$

By considering the variation of generalized Fibonacci quasi-periodic (GFQ) structures for $m=n$ named $\operatorname{GF}(m, n)$, it is mentioned that the whole band gaps are shifted to lower frequencies for increasing two parameters of the generalized Fibonacci sequence which is clearly shown in Fig. 4(a).

Thus, the amplitude of oscillations from cutoff frequencies around the band gaps decreases gradually.

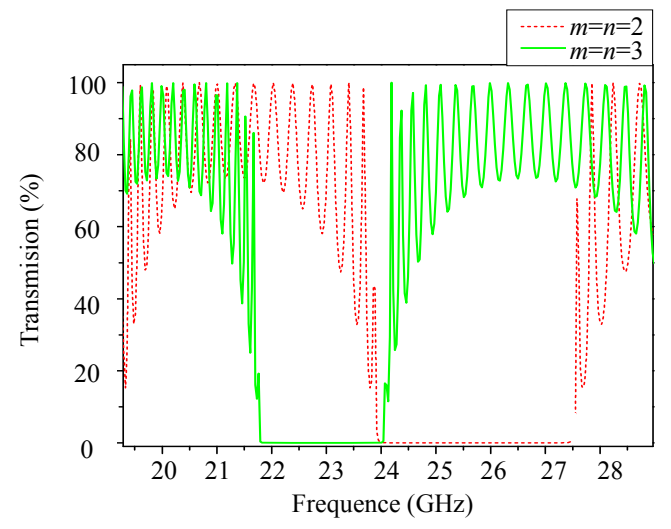

(a)

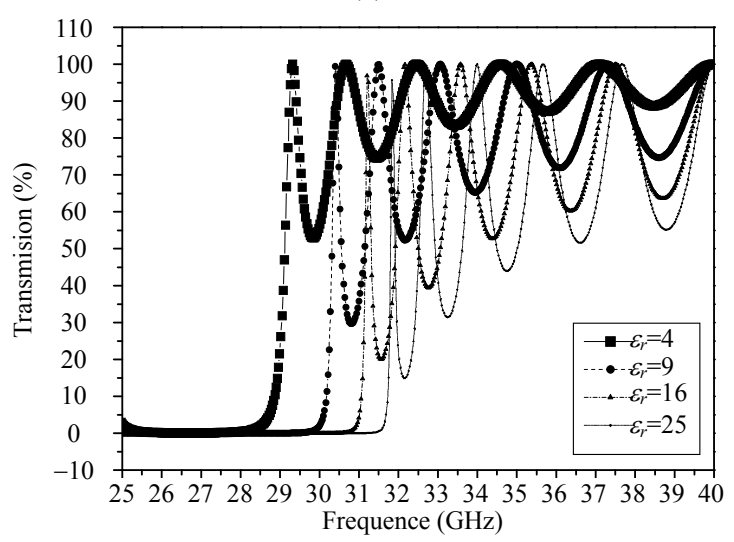

(b)

Fig. 4 Schematic representation of the shift of each band gap from the transmission spectrum of the generalized Fibonacci sequence versus the parameter of Fibonacci sequences (a) and the permittivity of the high refractive index (b).
Next, we increased the contrast index of multilayer's stacks by changing the permittivity of the high refractive index. Respectively, we noted the broadening and shift of the photonic band gaps monitored by a decrease in oscillations around it. As one can see from Fig. 4(b), here, there are sets of peaks appearing at equi-distance intervals, which are equivalent to sets of corresponding Bragg frequencies. The amplitude of each set decreases with an increase in frequencies $(\mathrm{GHz})$. Then, with an increase in the generation number, the band gaps gradually draw together until at transmission band emerges with some transmission peaks around the central frequency. We noted also an increase in the peak density producing a dark continuum at the edges of the curves.

For high order iterations, the undesired oscillations around the pseudo band gaps called "Kiessig fringes" are too high. There are various techniques suggested able to reduce the intensities of side lobes through the transmission spectrum similar to the technique of optimization: smoothing and apodization. The combination of the two techniques permits to reduce completely the whole side lobes and reduce the Kiessig fringes up to $95 \%$, able to eliminate the parasite information and limit ourselves to study only the principal part of the signal.

Figure 5 shows typically the transmission coefficient from the Thue-Morse multilayer stack for different multiplicity degrees $p$. One finds also a succession of pseudo band gaps of the low size which increases with the parameters of the generalized Thue-Morse sequence. Also, the center of each pseudo band gap varies according to the parameters $m$ or $n$ and the distribution of the layers $\mathrm{H}$ and $\mathrm{L}$ forming the system. According to the proposed method, the spectral contents of TM $(m, n)$ include multi narrowband gaps multiplied by a quasi-periodic envelope function as shown in Fig. 5.

The number of multi narrow band gaps within 
periods of the envelope function can be controlled by varying $p$, the degree of the multiplicity which joins $m$ to $n$. In Fig. 5, one can clearly see an important characteristic of the quasi-periodic Thue-Morse string which is the intrinsic special local symmetric repetition layer which forms the whole system. Actually, the fractal profile of the structures leads to a transmission spectrum with self-scaling properties, i.e. the variation of the spectrum with the frequency at each higher period is a modulated version, which is associated with the previous step.

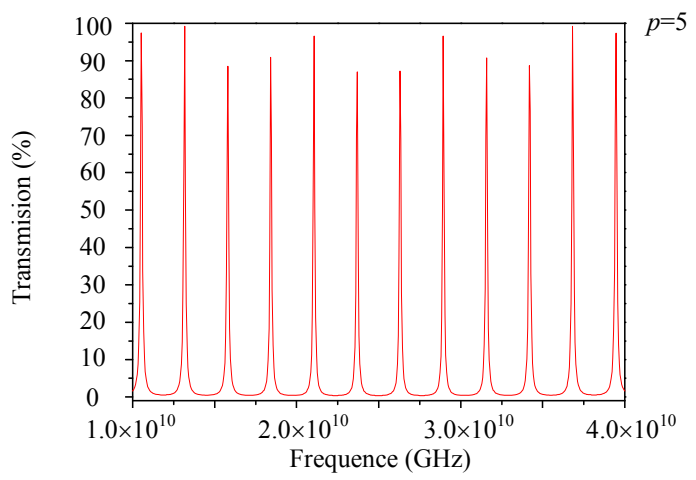

(a)

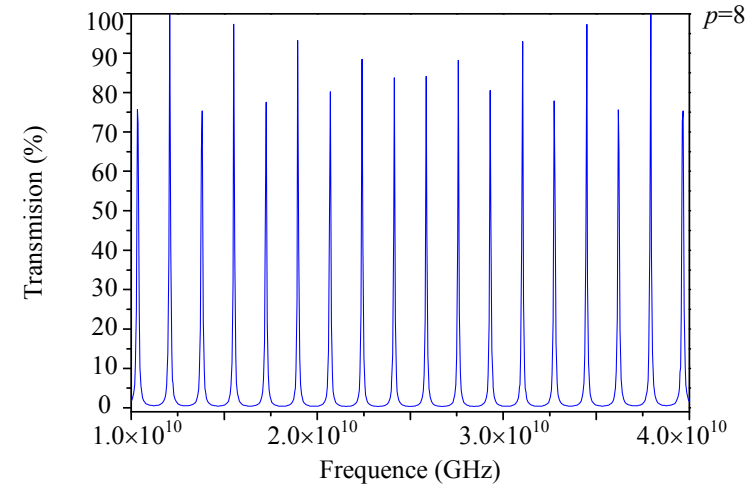

(b)

Fig. 5 Evolution of Thue-Morse (TMS) transmission spectra versus frequency $(\mathrm{GHz})$ for two multiplicity degrees, $p$ : (a) $p=5$ and (b) $p=8$.

In Fig. 5, the band gap centered at $25 \mathrm{GHz}$ (the center of the entire studied domain) is surrounded by the same number of gaps in the left and in the right. Also, this number corresponds to the value of the multiplicity degree $p$.

The hybrid quasi-periodic photonic band gaps structures are built respectively by the combination of the periodic and generalized quasi-periodic lattices. The subsystem is called the hybrid multilayer structure. It may be called also the generalized quasi-periodic structure with the defect. The defect inserted in the middle of the hybrid structure is periodic or generalized quasi-periodic lattice (Thue-Morse, Fibonacci) and has the same size as the basic cell of the quasi-periodic photonic.

We distinguished four types of hybrid photonic distributions where the first are based on the Fibonacci cell, and the second are based on the Thue-Morse cell named as follows:

- GF $(m, n) /$ Periodic/ GF $(m, n)$

- $\mathrm{GF}(m, n) / \mathrm{TM}(m, n) / \mathrm{GF}(m, n)$

- $\operatorname{TM}(m, n) /$ Periodic/ $\operatorname{TM}(m, n)$

$-\mathrm{TM}(m, n) / \mathrm{GF}(m, n) / \operatorname{TM}(m, n)$

The considered distribution of hybrid photonic band gaps named $\operatorname{GF}(m, n) / \operatorname{Periodic} / \mathrm{GF}(m, n)$ is essentially composed of two quasi-periodic cells arranged according to the 5th order of the generalized Fibonacci sequence called $\operatorname{GFC}(5,5,5)$. The high and low refractive indices of layers are taken as $n_{H}=3.13$ (Rogers duroid 6010) and $n_{B}=1$ (air), respectively.

The two cells aperiodic sequences are separated by a defect whose number of layers equals that of the basic quasi-periodic $\operatorname{GFC}(5,5)$ cells.

Figure 6 shows the transmission coefficient $T(\%)$ as the function of frequencies $F(\mathrm{GHz})$. In Figs. 6(a) and 6(b), we have two Fibonacci structures separated by periodic (40) and Thue-Morse (455) strucutres, respectively. Figure 6 shows that the transmission spectra, the size and the depth of the PBGs depend on the parameters $m$ and $n$ of the generalized structures.

Moreover, the variation and the distribution of layers lead to the displacement of the area of the Bragg reflection and the significant widening of the bandwidth. In addition, as a consequence of the fractal property of the displacement of layers, these structures exhibit a certain number of transmission 
peaks inside the frequency band gaps.

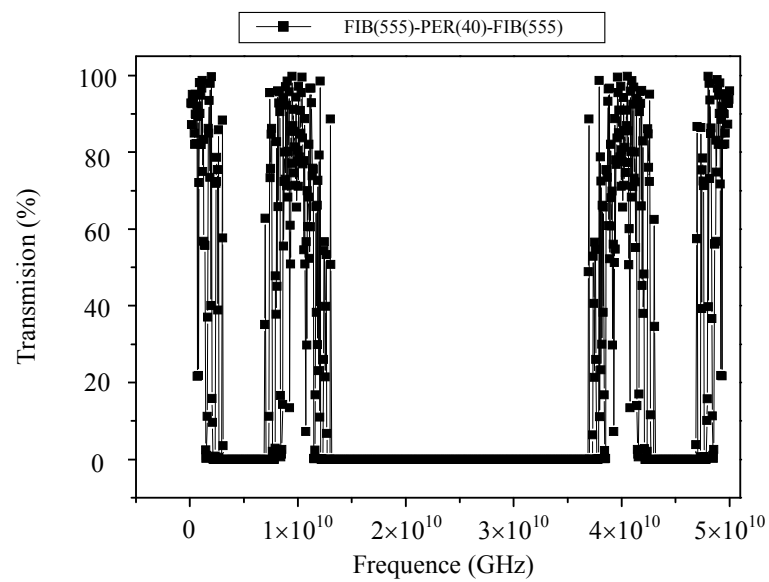

(a)

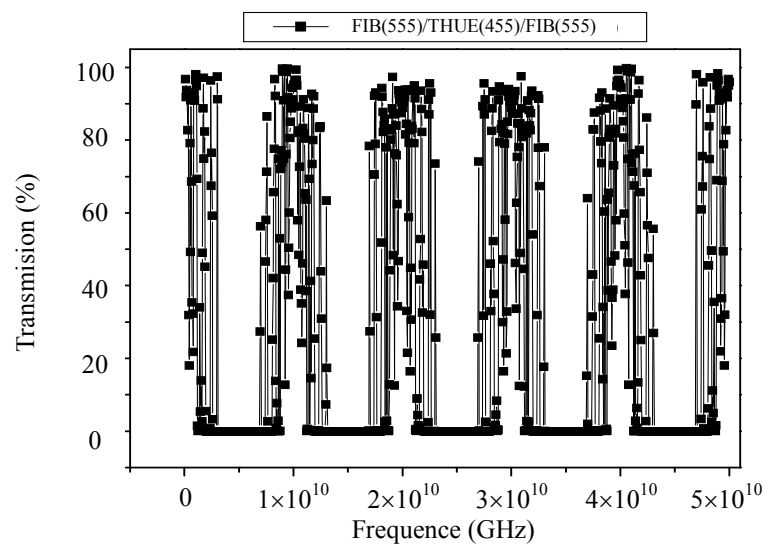

(b)

Fig. 6 Transmission spectra of two shapes of hybrid generalized Fibonacci sequences: (a) $\mathrm{GF}(m, n) / \mathrm{Periodic} / \mathrm{GF}(m$, $n)$ and (b) $\operatorname{GF}(m, n) / \operatorname{TMS}(m, n) / \mathrm{GF}(m, n)$ for $m=n=k=5$.

Figure 7 illustrates the transmission coefficient $T(\%)$ as a function of frequencies $(\mathrm{GHz})$ for two defects (periodic form and Fibonacci form). As seen from Fig. 7, the number of pseudo PBGs increases compared to the pseudo band gaps appeared from the periodic defect. In fact, the modulate variation of two types of layers is necessary to establish the band gaps. As mentioned above, the presence of the periodic lattice generates a large photonic band gap. As shown above, the frequency center changes the tendency of the type of the quasi-periodic structure and is not related to the period of the photonic quasi-crystal, but to the relative dielectric constant. This occurs because the high degree of rotational symmetry of the quasi-periodic structure and the weedy long-range order of the basic structural unit lead to different Bragg-like scattering effects inside the unit cell structures.

After presenting the microwave filtering property of quasi-crystals (Fibonacci or ThueMorse), we changed the type of defects inserted when the number and width of all layers $l$ varied, so a stacking of successive pseudo band gaps appeared, and more reflection peaks were obtained due to an increasing in the width of the layer $l$.
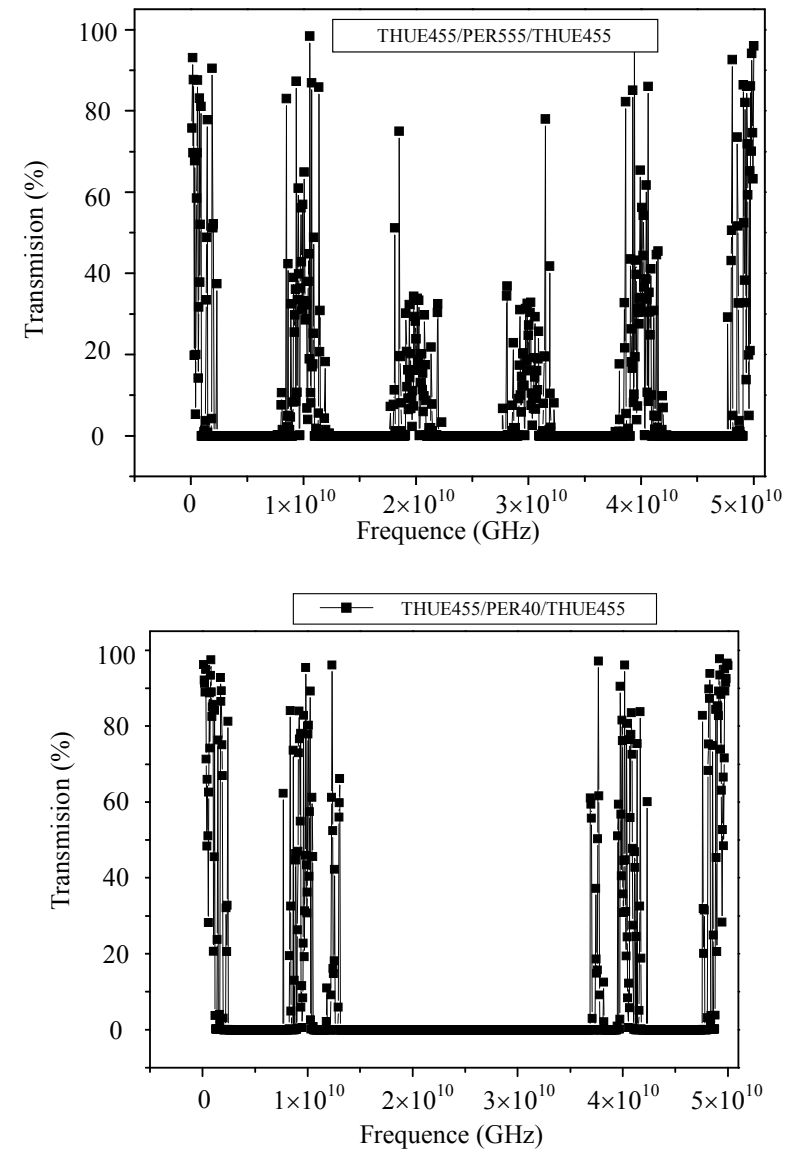

Fig. 7 Transmissions spectra of two forms of hybrid generalized Thue-Morse sequences TMS for $\mathrm{TM}(m, n) /$ Periodic/TM $(m, n)$ and $\operatorname{TM}(m, n) / \operatorname{GF}(m, n) / \operatorname{TM}(m, n)$.

Essentially, it is shown that each splitting peak open into transmission spectra is associated with the pseudo Brillion zone from hybrid quasi-periodic lattices. In addition, obviously the majority of the peaks for the pure periodic form are minor compared to the Fibonacci or Thue-Morse quasi-periodic arrangements. In fact, with an 
increase in the period, more peaks of the perfect transmission appear. The appearance of these sub stop band gaps is desired from the scope of their linear characteristics as a combination reflector for all-microwave telecommunications.

\section{Conclusions}

The transmission spectra of the Fibonacci and Thue-Morse multilayer structures in the microwave region were investigated. We extracted the transmission coefficient versus the parameters and the variable of the Thue-Morse sequence. Multi narrow band gaps cover all the frequency domains: 0-50 GHz. We found that the two sequences: Fibonacci or Thue-Morse, could exhibit multiple reflections of frequencies versus the multiplicity degree which joined the two parameters of the quasi-periodic system, $m$ and $n$. The position and number of such mini-pseudo bands depended on the number of the layer and the form of the distribution of two layers which consisted of the quasi-periodic multilayered structure. Also, we demonstrated that the defect mode transmission coefficient varied with the type of the defect layer. The number of multi narrow band gaps within could be controlled by varying the parameters $m$ or $n$. By increasing the photonic band gaps number, we could open a way towards achieving an interesting technological deivces such as a wave reflector component working in the microwave domain, $10 \mathrm{GHz}-40 \mathrm{GHz}$.

Open Access This article is distributed under the terms of the Creative Commons Attribution License which permits any use, distribution, and reproduction in any medium, provided the original author(s) and source are credited.

\section{References}

[1] D. Packiaraj, K. J. Vinoy, and A. T. Kalghatgi, "Analysis and design of a compact multi-layer ultra wide band filter," Progress In Electromagnetics
Research C, vol. 7, pp. 111-123, 2009.

[2] S. H. Yi, L. Z. Yuan, and G. B. Yuan, "Defect modes in multiple-constituent one-dimensional photonic crystals examined by an analytic bloch-mode approach," Chinese Physics Letters, vol. 22, no. 2, pp. 365-368, 2005.

[3] S. Golmohammadi, M. K. Moravvej-Farshi, A. Rostami, and A. Zarifkar, "Spectral analysis of Fibonacci-class one-dimensional quasi-periodic structures," Progress in Electromagnetics Research, vol. 75, pp. 69-84, 2007.

[4] J. W. Dong, P. Han, and H. Z. Wang, "Broad omnidirectional reflection band forming using the combination of Fibonacci quasi-periodic and periodic one-dimensional photonic crystals," Chinese Physics Letters, vol. 20, no. 11, pp. 1963-1965, 2003.

[5] A. Barriuso, J. Monzon, L. Sanchez-Soto, and A. Felipe, "Comparing omnidirectional reflection from periodic and quasiperiodic one-dimensional photonic crystals," Optics Express, vol. 13, no. 11, pp. 3913-3920, 2005.

[6] Y. Akahane, M. Mochizuki, T. Asano, Y. Tanaka, and S. Noda, "Design of a channel drop filter by using a donor-type cavity with high-quality factor in a two-dimensional photonic crystal slab," Applied Physics Letters, vol. 82, no. 9, pp. 862-865, 2007.

[7] W. J. Hsueh, S. J. Wun, Z. J. Lin, and Y. H. Cheng, "Features of the perfect transmission in Thue-Morse dielectric multilayers," Journal of the Optical Society of America B, vol. 28, no. 11, pp. 2584-2591, 2011.

[8] F. Qiu, R. W. Peng, X. Q. Huang, X. F. Hu, M. Wang, A. $\mathrm{Hu}$, et al., "Omnidirectional reflection of electromagnetic waves on Thue-Morse dielectric multilayers," Europhysic Letter, vol. 68, no. 5, pp. 658-663, 2004.

[9] Enrique Macia, "Exploiting aperiodic designs in nanophotonic devices," Reports on Progress in Physics, vol. 75, no. 3, pp. 036502, 2012.

[10] C. H. O. Costa, M. S. Vasconcelos, P. H. R. Barbosa, and F. F. Barbosa Filho, "Fractal spectra in generalized Fibonacci one-dimensional magnonic quasicrystals," Journal of Magnetism and Magnetic Materials, vol. 324, no. 14, pp. 2315-2323, 2012.

[11] H. X. Ding, Z. H. Shen, X. W. Ni, and X. F. Zhu, "Multi-splitting and self-similarity of band gap structures in quasi-periodic plates of Cantor series," Applied Physics Letters, vol. 100, no. 8, pp. 083501, 2012.

[12] X. Jiang, Y. Zhang, S. Feng, K. C. Huang, Y. Yi, 
and J. D. Joannopoulos "Photonic band gaps and localization in the Thue-Morse structures," Applied Physics Letters, vol. 86, no. 20, pp. 201110-1201110-3, 2005.

[13] Z. Li, "Principles of the plane-wave transfer-matrix method for photonic crystals," Science and Technology of Advanced Materials, vol. 6, no. 7, pp. 837-841, 2005.

[14] G. Guida "Numerical studies of disordered photonic crystals," Progress in Electromagnetics Research, vol. 41, pp. 107-131, 2003.

[15] M. Teimourpour, "Nonreciprocal bistability in nonlinear fractal Fibonacci photonic crystals,"
Journal of Optics, vol. 14, no. 3, pp. 035501, 2012.

[16] S. Mingaleev and Y. Kivshar, "Nonlinear transmission and light localization in photonic crystal waveguides," Journal of the Optical Society of America B, vol. 19, no. 9, pp. 2241-2249, 2002.

[17] V. Grigoriev and F. Biancalana, "Bistability, multistability and nonreciprocal light propagation in Thue-Morse multilayered structures," New Journal of Physics, vol. 12, no. 5, pp. 053041, 2010.

[18] P. Yeh and A. Yariv, Optical waves in crystals. New York: John Wiley \& Sons Group, 1984, p.589. 